\title{
COP: A New Method for Extracting Edges and Corners
}

\author{
Sun Cheol Bae, In So Kweon \\ Dep. of Automation and Design Eng., Korea Advanced of Institute of Science and Technology. \\ 207-43, Cheongryangri-dong, Dongdaemoon-gu, Seoul, KOREA. \\ Internet Address:iskweon@cais.kaist.ac.kr TEL. +82-2-958-3465, FAX. +82-2-960-0510
}

\begin{abstract}
This paper presents a new, simple and effective low level processing method to obtain features such as edges and corners. In both edge and corner extraction algorithms, we use two oriented cross operators called COP (Crosses as Oriented Pair). To obtain edges, many conventional edge operators use derivative convolution masks and are followed by the conventional nonmaximum suppression algorithm which needs rather complicated edge direction calculation. Moreover, most conventional derivative-based operators suffer from poor connectivity at junctions, sensitivity to noise and two extrema when they are applied to a line. But COP makes it possible to find edge direction very easily, to localize edge position accurately and to obtain connected edges. Second, we propose a new corner detection algorithm using COP. Most conventional corner detectors have shortcomings such as missing junctions, poor localization, sensitivity to noise and high computational cost. With the characteristics of COP and simple rules, we can accomplish a very fast, accurate and robust corner detection than any other corner detector. Performances of two proposed algorithms are described with test results.
\end{abstract}

\section{Introduction}

Edge detection has been one of the most active fields in computer vision. Most of previous research on edge detection have been based on discrete approximations to differential operations. Recently, Smith and Brady [1] proposed a new low-level processing "SUSAN", which uses simple masking operations, namely, only counts the number of pixels dissimilar to the center pixel of a circular window. So it is very robust to noise. Most of the edge detectors use conventional non-maximum suppression algorithm to obtain meaningful edges. But it often produces undesirable effects such as thick and broken edges or bad localized edges.

The recent literature on corner detection can be widely divided into two approaches. The first approach is to initially segment the image into regions, represent the object boundary with a chain code, and then search for points having maximum curvature or perform a polygonal approximation on the chains and then search for the line segment intersections. The major drawback of this approach is that the performance of corner detection depends on the preceding image segmentation result. To overcome this, most of recent attempts use the second approach, which mainly uses gradients and surface curvatures $[1,2,3,4,5,6,7,8]$. This approach, however, also suffers from localization of corners and noise. Smith and Brady [1] use a circular window, composed of 37 pixels, to obtain USAN area whose centroid is then used to suppress false positives. Finally, non-maximum suppression finds corners. The corner localization and noise robustness are good or better than other algorithms. But it takes much computational cost in obtaining USAN area and finding corners due to the size of the window. In some cases, they also produce false positive and negative corners. 


\section{COP (Crosses as Oriented Pair)}

In this section, we describe the characteristics of COP which is composed of two 3 by 3 oriented crosses as shown in Figure 1.
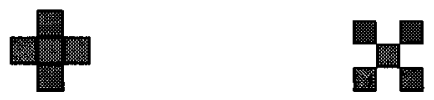

Figure 1. Two crosses of COP.

Each of COP has a very interesting property. The left one responds strongly to $\pm 45^{\circ}$ edges, and the right one to the horizontal and vertical edges. Figure 2 shows these characteristics of each of COP with simple images. Each response shows the inverted USAN area, namely, the number of pixels dissimilar to the mask's nucleus. Moreover, each of COP shows peak values near the corners. Another important characteristic is that COP makes it possible to determine orientation in a very simple manner. Briefly speaking, if the inverted USAN output of the left cross is larger than that of the right one, edge direction is $+45^{\circ}$ or $-45^{\circ}$. In this case, if sum of the inverted USAN output of top-left and bottom-right neighbors is larger than that of top-right and bottom-left ones, then edge direction is $-45^{\circ}$ and vice versa. If the inverted USAN output of the right cross is larger than that of the left one, edge direction is $0^{\circ}$ (horizontal) or $90^{\circ}$ (vertical). In this case, if sum of the inverted USAN output of left and right neighbors is larger than that of top and bottom ones, then edge direction is $0^{\circ}$ and vice versa. Other cases which are not mentioned in the above happen at corners or some places where orientation changes.

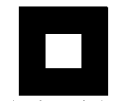

(a) original image (b) original image

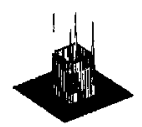

(c) output of each cross for image (a).

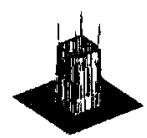

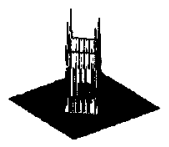

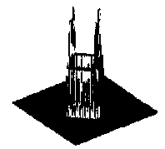

(d) output of each cross for image (b).

Figure 2. Each response of COP to simple synthetic images.

\section{Edge extraction}

\subsection{Algorithm}

Our edge detection algorithm performs the following five steps.

Step 1. Obtain inverted USAN area, R1 and R2, using COP (R1: output of R2: output of );

Step 2. Calculate edge direction;

Step 3. Discard the elements having lowest edge response (when $\mathrm{R} 1 * \mathrm{R} 2<2$ );

Step 4. Apply non-maximum suppression; and

Step 5. Edge refinement is added if needed. 


\subsection{Experimental results}

Experiments are carried out for several synthetic and real images. Figure 3 shows the responses of COP to some synthetic images including multi-region junctions (" $T$ ", "Y", "X"). All outputs of COP show good performances. Some experiments are carried out on real scenes. COP produces satisfactory results as shown in Figure 4. One complicated outdoor road scene is also used to test COP edge detector. Some problems such as fluctuating edges take place in this case due to noise. But this may be cured if refining process is fulfilled.
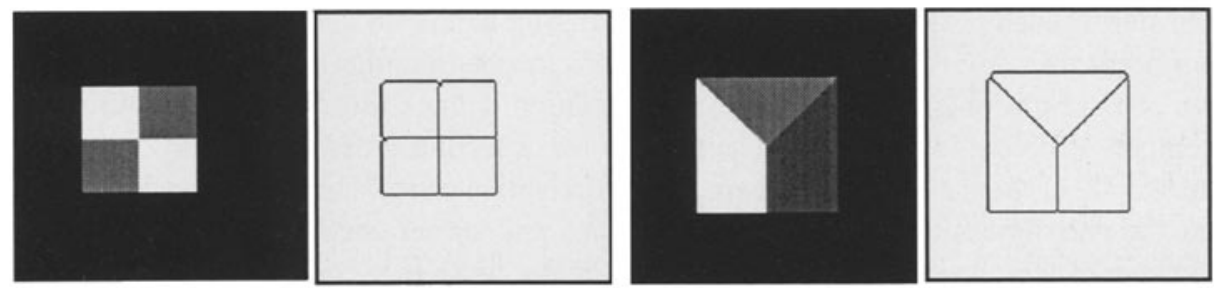

Figure 3. Outputs of COP for synthetic images.
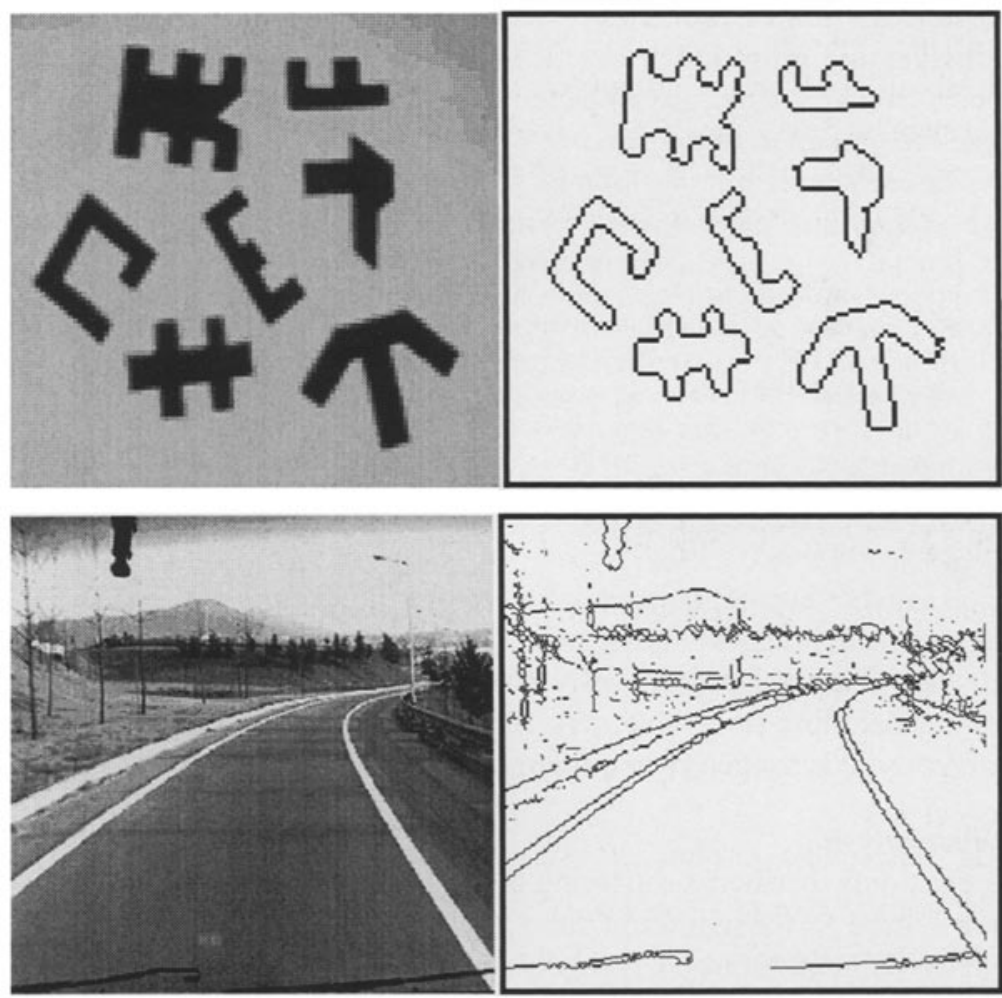

Figure 4. Outputs of COP for real images. 


\section{Corner extraction}

Our corner detection algorithm is mainly divided into the following five steps:

Step 1. Obtain inverted USAN area using COP;

Step 2. Obtain corner candidates;

Step 3. Compare each output of COP to obtain useful directional information;

Step 4 . Calculate $2^{\text {nd }}$ order tangential derivatives of original intensity value among the candidates obtained in step 2 except the predetermined corners; and

Step 5. Find local maximum in a search window.

In step 1, each output of $C O P$ has values ranging from 0 to 4 . In step 2, we perform our algorithm only when $[R 1(i, j)+R 2(i, j)]>3$ to obtain corner candidates. In step 3 , we can simply obtain edge direction information using COP. All we have to do is compare each output of COP. In step 4 , we use a second order directional derivative mask, $[-2,-1,6,-1,-2]$, to obtain tangential derivative of intensity. This mask represents the one dimensional Laplacian of Gaussian and corner checking rules are applied to some of the obtained corner candidates. Finally, in step 5, corners are identified by taking local maxima in the search window.

\subsection{Corner candidates and rules}

We consider any point in the image as corner candidate if each inverted USAN output of COP, $R 1$ and $R 2$, satisfies the condition that $R 1+R 2>3$ at that point. To overcome the limitation of the size of COP and make full use of the characteristics of $\mathrm{COP}$, we define simple rules as follows.

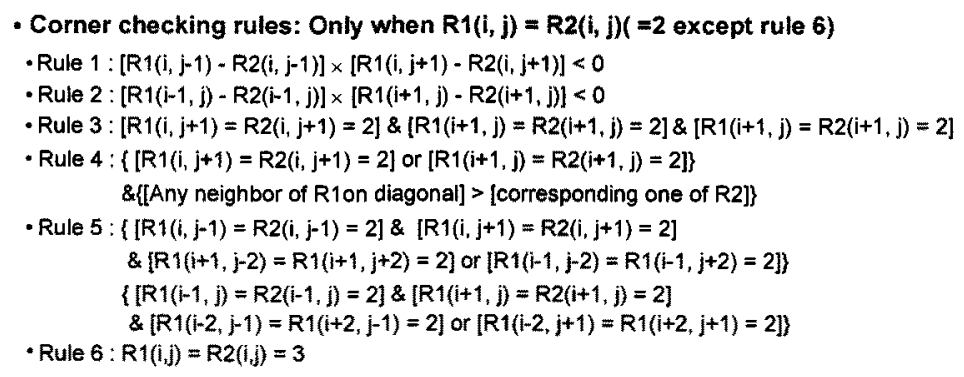

If a pixel satisfies any of six rules, we select that point as corner. Rules 1 and 2 mean that there exist two edges whose directions are changing in the horizontal and the vertical sides of a pixel, respectively. Rules 3 and 4 are similar to rules 1 and 2, but used to consider more smooth corners. Junctions are also considered in rule 5. Rule 6 is clear because this happens when sharp corner exists.

\subsection{Rule analysis}

There exist only 7 patterns satisfying the case that $R 1$ and $R 2$ are all equal to 2 , as shown in Figure 5. We avoid the duplicated cases such as the axis-symmetric and rotationally symmetric ones. Especially, the first pattern is important and should be well analyzed because this case has the highest possibility of occurrence. In Figure 6, we analyze this case in detail. In Pattern A, R1 is larger than R2 at left neighbor, which means that there may be $+45^{\circ}$ or $-45^{\circ}$ edge locally. In this case, as you know, 
$+45^{\circ}$ edge exists. And $\mathrm{R} 2$ is larger than $\mathrm{R} 1$ at right neighbor, which means that there may be locally horizontal or vertical edge. In this case, horizontal edge exists. Anyway the center point is regarded as corner point by rule 1 . But rule 1 is also applied to the case like pattern D. By rules, pattern B is not considered as corner. Look at the pattern $\mathrm{C}$, in this case the center pixel and its right neighbor may be regarded as noises on flat edge, or may be corners if significant orientation change happens in the right part of this pattern. We also consider this case by rule 5 . There are many other patterns satisfying the case that $R 1$ and $R 2$ are all equal to 2 . Such patterns are also checked whether they are corners or not by proposed corner checking rules.

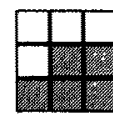

(1)

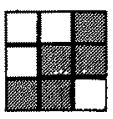

(2)

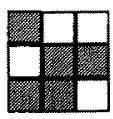

(3)

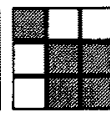

(4)

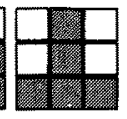

(5)

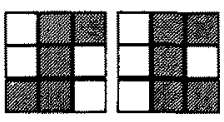

(6)

(7)

Figure 5. Typical seven patterns when R1 and R2 are all equal to 2 .

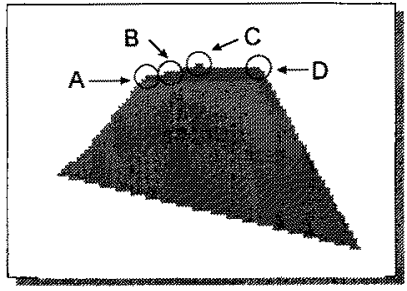

(a) Input image
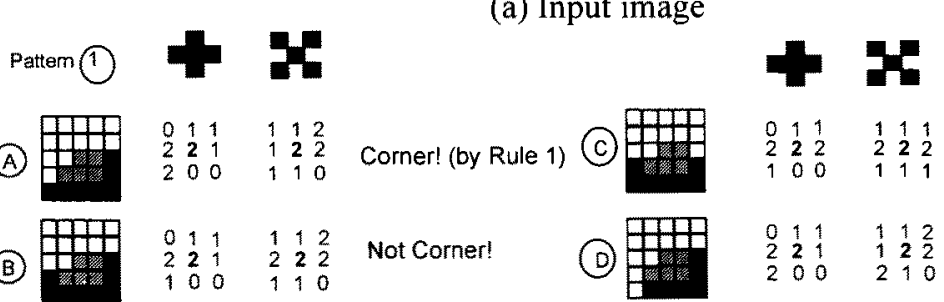

Indeterminate but, it may be Corner! (by rule 5)

Not Corner:

(b) Detailed patterns of areas circled in (a)

Figure 6. Rule analysis for a synthetic image.

\subsection{Experimental results}

The results of testing this corner algorithm are shown and discussed for several synthetic and real images. Some images are used to test its sensitivity to noise. Finally, one example is added to test how flexible our comer finder is, as threshold varies. All images are tested to compare COP' s performance with SUSAN. For fair comparison, we use original SUSAN algorithm supported by Internet. Our algorithm produces satisfactory results for all cases. Results for real images are shown in Figures 7 8. Figure 9 shows the output of two corner finders for one real image as the threshold value varies from 30 to 60 in 10 increments. If the threshold value is lower than some optimal threshold, false positives show up. In the opposite case, false negatives show 
up. As you see, the performance of the COP corner detector doesn't degrade so much as the threshold value varies. With respect to speed, COP only needs small 3 by 3 masking operations. Therefore, time complexity is at least 4 times lower than SUSAN. Simplicity in orientation calculation is another factor to shorten the computation time. For example, it took at most $0.2 \sim 0.3$ seconds to process 256 by 256 real scene on a 586 PC.
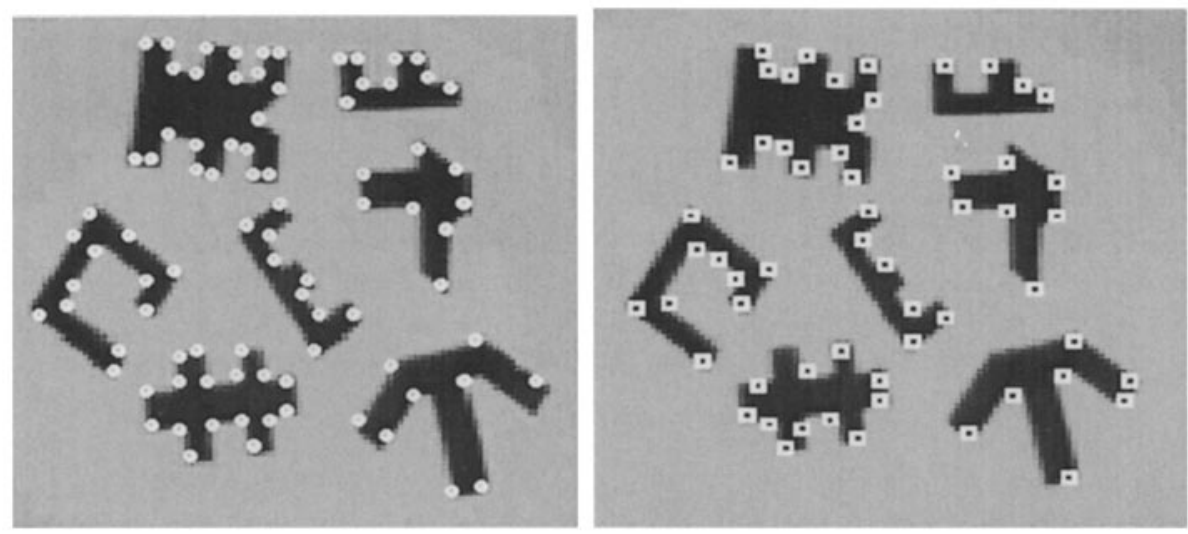

Figure 7. Outputs of COP (left) and SUSAN (right).

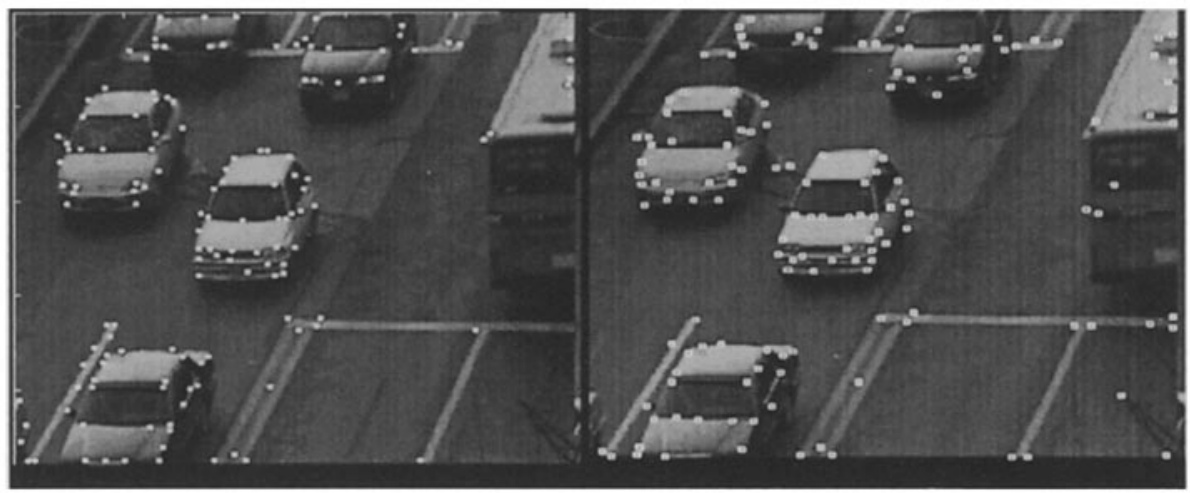

Figure 8. Outputs of COP (left) and SUSAN (right).

\subsection{Comparison of each corner detector}

In this section, we briefly summarize the performance of each detector for some images only in view of detectability. Figure 10 summarizes the results of each corner detector to a varing Gaussian smoothing factor $\sigma$ whose value is $0.5,1.0$ and 1.5 , respectively. COP has no false positives and less false-alarms. In Table 1, all numbers denoting false-positives and negatives are expressed relatively to the number of real corners. Though more false-positives than SUSAN, COP has less false-negatives, which means good detection. In this test, we use a five by five search window to find local maxima in each corner detector. 


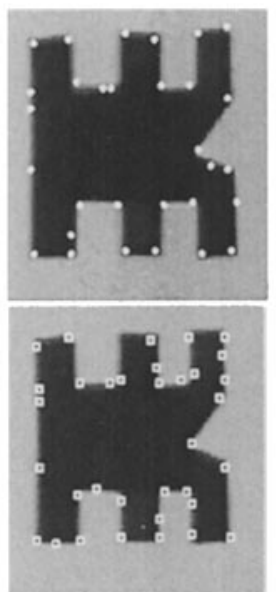

(a) $\mathrm{Th}=30$

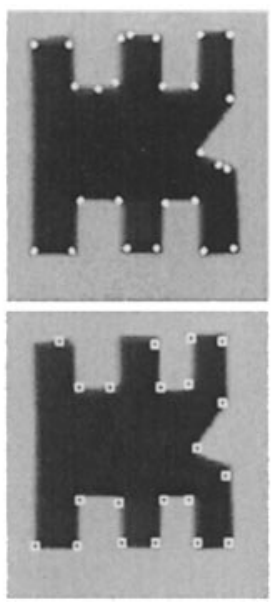

(b) $\mathrm{Th}=40$

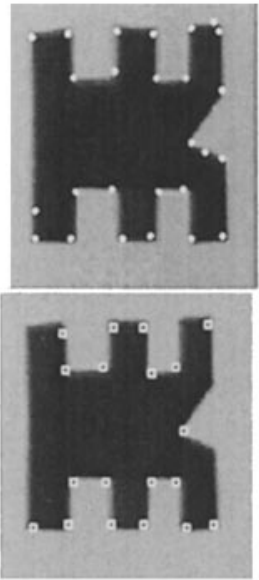

(c) $\mathrm{Th}=50$
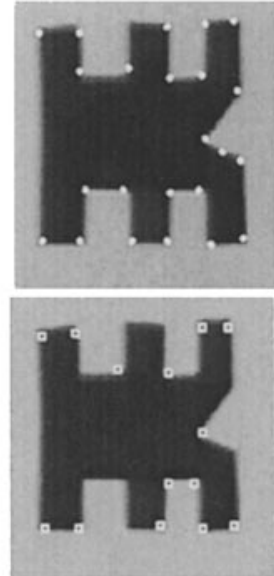

(d) $\mathrm{Th}=60$

Figure 9. The threshold flexibility test of COP (top row) and SUSAN (bottom row).
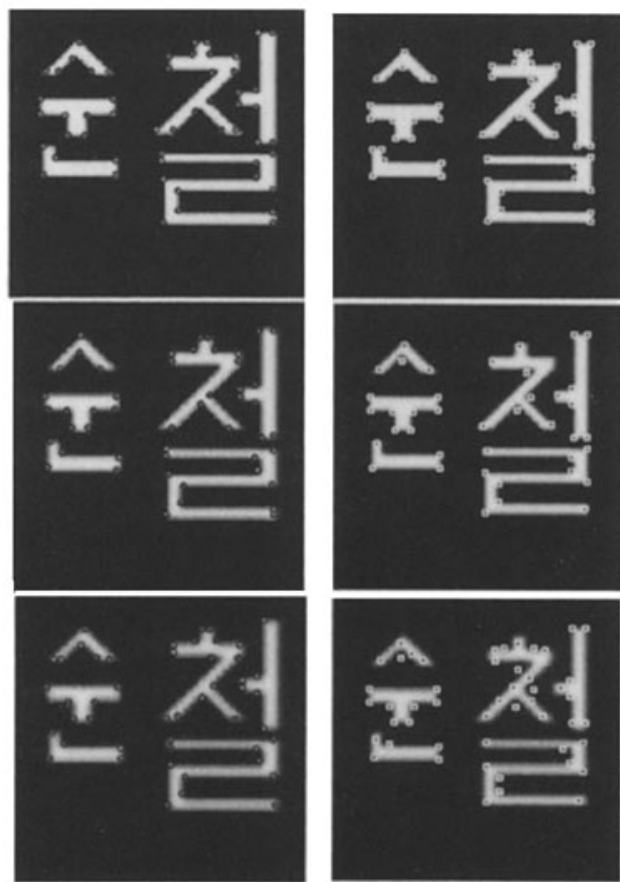

(a) Outputs of COP (left) and SUSAN (right)

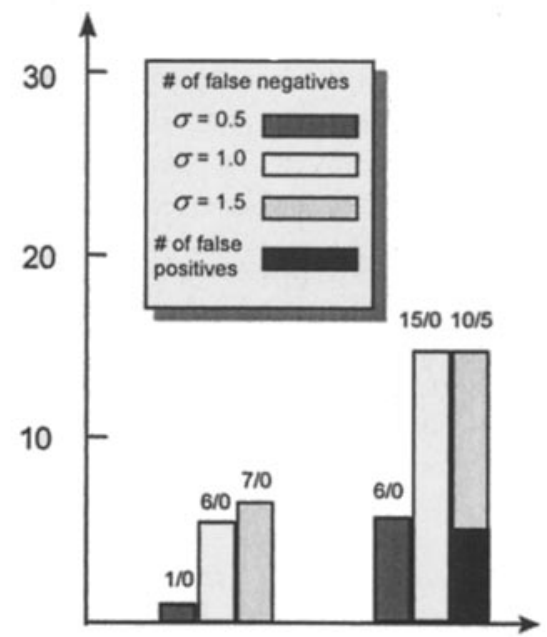

(b) Number of false alarms

Figure 10. Detectability comparison of each corner detector with a varying Gaussian smoothing factor, $\sigma$ ranging from 0.5 to 1.5 in 0.5 increments. 
Table 1. Detectability comparison of each corner detector.

\begin{tabular}{|c|c|c|c|}
\hline \multicolumn{2}{|c|}{$\begin{array}{l}\text { Test talse detector } \\
\text { image }{ }^{2} \text { alarm }\end{array}$} & COP & SUSAN \\
\hline \multirow{2}{*}{ 순절 } & $+\ldots$ & $0 / 55$ & $0 / 55$ \\
\hline & - & $0 / 55$ & $0 / 55$ \\
\hline \multirow{2}{*}{$\boldsymbol{A}_{\mathrm{H}}$} & + & $0 / 33$ & $0 / 33$ \\
\hline & - & $0 / 33$ & $5 / 33$ \\
\hline \multirow{2}{*}{ sid } & + & $3 / 95$ & $1 / 95$ \\
\hline & - & $12 / 95$ & $38 / 95$ \\
\hline \multirow{2}{*}{ 目蛋 } & + & $0 / 70$ & $0 / 70$ \\
\hline & - & 0170 & $1170^{\circ}$ \\
\hline \multirow{2}{*}{ total } & + & $3 / 253$ & $1 / 253$ \\
\hline & - & $12 / 253$ & $44 / 253$ \\
\hline
\end{tabular}

\section{Conclusions}

In this paper, we proposed two oriented cross operators, COP, which provide a useful information to extract low-level features due to its characteristics, preference for different edges and simple orientation determination. With the help of COP, we proposed two new feature detection algorithms, for edges and corners. In edge detection algorithm, we don't use the conventional non-maximum suppression algorithm but use a new non-maximum suppression rules. In complicated outdoor scene, there remains some problems such as fluctuating and broken edges like other edge extraction algorithms though it gives good results for synthetic and some real images. However, COP "corner detector" provides satisfactory results for all synthetic and noisy real scenes. Its small size lowers computational cost and increases localization accuracy. Moreover, its simple orientation selection and noise robustness make it possible to produce very effective and good performance.

\section{References}

[1] S. M. Smith and J.M. Brady, "A new Approach to Low Level Image Processing", IJCV, 1996. In publication.

[2] P. R. Beaudet, "Rotational invariant image operators", ICPR pp579-583,1978.

[3] L. Kitchen and A. Rosenfeld, "Gray-level corner detection", P. Recognition Letters, 1, 1982.

[4] L. Dreschler and H.-H. Nagel, "Volumetric Model and 3D Trajectory of a Moving Car Derved from Monocular TV-frame Sequence of a Street Scene", CVGIP, 20(3):pp 199- 228, 1981.

[5] C.G. Harris and M. Stephens, "A combined corner and edge detector", In $4^{\text {th }}$ Alvey Vision Conference, pp147-151,1988.

[6] R. Deriche and G. Giraudon, "A computational Approach for Corners and Vertex Detection ", UCV, 10(2):pp101-124, 1993.

[7] H. Wang and M. Brady, "Real-time corner detection algorithm for motion estimation", 13(9): pp 695-703, 1995.

[8] C. H. Chen, J. S. Lee and Y. N. Sun, "Wavelet transformation for gray-level comer detection", 28(6), pp853-861, Pattern Recognition, 1995.

[9] J. F. Canny, "Finding edges and lines in images", Master's thesis, MIT, Cambridge, USA, 1983. 